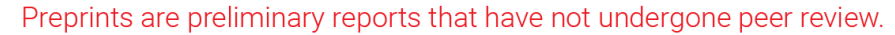 They should not be considered conclusive, used to inform clinical practice, or referenced by the media as validated information. \\ Feasibility study of a verification method for proton and carbon ion radiotherapy plan delivery accuracy check
}

jun zhao ( $\sim$ zhaojunphy@gmail.com )

Shanghai Proton and Heavy Ion Center,Fudan University Shanghai Cancer Hospital

\section{Zhi Chen}

Department of medical physics,Shanghai proton and heavy ion center

\section{Xianwei Wu}

Department of medical physics,Shanghai proton and heavy ion center

\section{Ying Xing}

Department of medical physics, Shanghai proton and heavy ion center

\section{Yongqiang Li}

Department of medical physics,Shanghai proton and carbon ion center

\section{Research}

Keywords: proton and carbon ion radiotherapy, scanning beam, plan verification, beam parameter deviation

Posted Date: March 25th, 2020

DOl: https://doi.org/10.21203/rs.3.rs-19264/v1

License: (c) (1) This work is licensed under a Creative Commons Attribution 4.0 International License. Read Full License 


\title{
Feasibility study of a verification method for proton and carbon ion radiotherapy plan delivery accuracy check
}

Jun Zhao ${ }^{1,2 \#^{*}}$, Zhi Chen ${ }^{3 \#}$, Xianwei $\mathrm{Wu}^{3}$, Ying Xing ${ }^{3}$, Yongqiang $\mathrm{Li}^{3}$

1. Department of Radiation Oncology, Fudan University Shanghai Cancer Center, Shanghai, China

2. Department of Oncology, Shanghai Medical College, Fudan University, Shanghai, China

3. Department of Medical Physics, Shanghai Proton and Heavy Ion Center, Shanghai, China

\# Jun Zhao and Zhi Chen are co-first authors.

*Corresponding author: Jun Zhao, email:zhaojunphy@gmail.com

Department of Radiation Oncology, Fudan University Shanghai Cancer Center, 270 Dongan Road, Xuhui, Shanghai, China 200032

\begin{abstract}
Background: All plan verification systems available for particle therapy are designed for pre-treatment verification. The plan delivery accuracy during treatment are unknown. The purpose of this study is to introduce a verification method and develop a software for proton and carbon ion plan delivery accuracy check.
\end{abstract}

Methods: A program was developed using Matlab to reconstruct dose from beam parameters recorded in log files and compare the dose reconstructed with the dose calculated by treatment planning system (TPS). Ten carbon ion plans and ten proton plans were enrolled in this study for algorithm validation, sensitivity analysis and plan delivery verification. The dose reconstruction algorithm was validated by comparing the dose calculated by TPS with reconstructed dose using the same beam parameters. The sensitivity 
of gamma pass rate to spot size deviation, position deviation and particle number deviation were analyzed by comparing dose reconstructed from pseudo plans which have manually added errors with original plan dose. Then plan delivery verification using homemade software were done for the 20 actual treated plans.

Results: A program for plan delivery verification was developed. For the validation of dose reconstruction algorithm, the mean dose difference between reconstructed dose and plan dose were $0.70 \% \pm 0.24 \%$ and $0.51 \% \pm 0.25 \%$ for carbon ion and proton plans, respectively. According to our simulation, the Gamma pass rate of carbon ion beam is more sensitive to spot position deviation and particle number deviation, and the Gamma pass rate of proton beam is more sensitive to spot size deviation. For the actual plan delivery verification using homemade software, the mean gamma pass rate were $99.47 \% \pm 0.48 \%$, $99.36 \% \pm 0.50 \%$ and $99.48 \% \pm 0.50 \%$ for carbon ion beams and $99.92 \% \pm 0.13 \%, 99.96 \%$ $\pm 0.06 \%$ and $99.89 \% \pm 0.13 \%$ for proton beams at three different depth of high dose region using $3 \mathrm{~mm} / 3 \%$ criteria.

Conclusions: A software was programed and the algorithm was verified. The method we introduced and the software we made for plan delivery verification is feasible and reliable. The verification method presented in this study can be easily repeated in other hospital.

Key words: proton and carbon ion radiotherapy, scanning beam, plan verification, beam parameter deviation

\section{Introduction}

Proton and carbon ion radiotherapy have gained great attention in recent years and have been used for different tumor sites ${ }^{1-4}$. Compared with photon radiotherapy, proton and 
carbon ion radiotherapy can provide a higher target dose while allowing better sparing of normal tissue due to the depth dose distribution, low entrance dose followed by the Braggpeak $^{5-7}$. In addition to the physical advantage of the depth dose profile, proton and carbon ion offer a differential relative biological effectiveness (RBE) with depth, due to a relatively low linear energy transfer (LET) in the entrance region with progressively increased LET at the peak. The physical characteristics of sharp dose peak at a particular range in tissue make the delivered dose distribution sensitive to delivery uncertainties, which could make the Bragg-peak located at a wrong position ${ }^{8,9}$. This may result in insufficient dose in the target region or severe side effects. Therefore, a quantitative verification of delivered dose distribution is desirable and important to ensure patient receive correct dose distribution during treatment in each fraction. Currently, all available plan verification methods were off-line and pre-treatment, using 3D pinpoint ion chambers blocks, ion chamber matrix or films measured in phantom ${ }^{10-13}$. We can't get delivered dose distribution during treatment with such solutions. The potential online verification methods are the positron emission tomography (PET) verification and prompt gamma ray verification ${ }^{14-17}$, which can only do range verification with not very good precision and far from fully clinical application.

All radiotherapy systems have log files to record the information during treatment. The proton and carbon ion radiotherapy system with pencil beam scanning used in our hospital has no exception. The log files recorded for each treatment fraction include the information of actual delivered spot position, spot size and particle number in each spot for each isoenergy slice (IES). All log-file data are measured by the beam monitoring system in the beam nozzle in front of patient, which represents the actual beam delivery to patients can 
be used to evaluate the delivered dose distribution. The purpose of this study is to introduce a verification method and develop a software for proton and carbon ion plan delivery accuracy check.

\section{Methods and materials}

\subsection{Beam Delivery System}

The Siemens ion beam delivery system used in this study has a synchrotron for acceleration and a modulated scanning beam technique paired with energy stacking for beam delivery. The accelerator can produce proton and carbon ion beams with energies range from 48.08 to $221.07 \mathrm{MeV} / \mathrm{n}$ and 86.22 to $430.12 \mathrm{MeV} / \mathrm{n}$, respectively. Each ion species can be accelerated and extracted at one of the 296 different pre-programmed energy levels, which corresponding to approximately $1 \mathrm{~mm}$ step of range in water. For each energy level, there are 5 different spot sizes. The spot sizes at iso-center in air are $8.10 \mathrm{~mm}$ to 32.65 $\mathrm{mm}$ and $3.38 \mathrm{~mm}$ to $13.53 \mathrm{~mm}$ for proton and carbon ion beams, respectively. Higher energy has smaller spot size. The modulated scanning technique make the beam remains at each spot until the requested monitor units is reached and then swiftly moves the beam to the next spot without turning off the beam. The ion beam delivery system has a dynamic intensity control system (DIC), which computes and adapts the extraction rate during the delivery of ions at each spot to make the efficient and accurate extraction. Beam delivery is monitored by the beam application and monitoring system (BAMS). Fig.1 shows the diagram of the BAMS. Beam parameters measured by BAMS are recorded in the log file. The BAMS in each treatment room consists of three transmission ionization chambers and two multi-wire proportional chambers. The ionization chambers are used to monitor the 
number of ions delivered at each spot. The multi-wire proportional chambers are used to monitor the spot position and size. The spot positions integrated with horizontal and vertical width are measured every $250 \mu$ s using multi-wire proportional counters, and the measuring accuracy is within $0.5 \mathrm{~mm}$. The flux is sampled by a transmission parallel plate ionization chamber at $1 \mu$ s time intervals, which can quickly turn the beam to next spot when reaching the prescribed particle numbers in one spot

\section{Fig.1}

\subsection{Software development}

In our plan delivery system, the nominal plan parameters from TPS are stored in physical beam plan (PBP). During patient treatment, the actual plan delivery parameters are measured by BAMS. The measured values at BAMS position are converted to values at iso-center according to the base data lookup table and then stored in the physical beam record (PBR). A homemade program was developed using Matlab to reconstruct the 2D dose distribution according to the beam parameters, such as the spot size, spot position, particle number in each spot, virtual source distance, depth dose distribution data and double Gaussian distribution parameters. Double Gaussian distribution model is used for lateral beam profile, which can calculate dose at halo area at large radial distance more accurate than single Gaussian ${ }^{18,19}$. Gamma analysis ${ }^{20}$ function was also programed to compare the difference between reconstructed dose and plan dose to check the accuracy of treatment delivery. Local normalization in the dose difference is used in gamma evaluation.

\subsection{D dose distribution reconstruction}

In this study, the dose was reconstructed in a cubic water phantom, which is used in 
TPS for calculating verification plan. Then we can compare reconstructed dose with TPS calculated dose. The dose at certain depth in water generated by a single pencil beam can be described as

$\mathrm{D}=N \cdot f\left(\sigma_{1}, \sigma_{2}, w_{1}, w_{2}, x, y, z\right) \cdot D D D\left(E_{\text {beam }}, z\right)$

$N$ is the total number of delivered particles. Lateral beam profile is described by double Gaussian distribution $f\left(\sigma_{1}, \sigma_{2}, w_{1}, w_{2}, x, y, z\right)$, which is the function of beam position and depth in water. $\sigma_{1}, \sigma_{2}, w_{1}$ and $w_{2}$ are the width and weight of the first and second Gaussian. The double Gaussian distribution parameters are stored in the base-data of our TPS as lookup table as functions of depth in water for the whole required energy spectrum E. The lateral beam cut-off is 3.5\%. $x$ and $y$ are the position in $\mathrm{x}$ and $\mathrm{y}$ direction at depth $z$ in water. $D D D\left(E_{\text {beam }}, z\right)$ is the depth dose value at depth $z$ in water for the beam with energy $E_{\text {beam }}$. The total $2 \mathrm{D}$ dose distribution in water at certain depth is an integral of dose at the depth contributed by all beams including the fragmentation tail pass through the slice. So the 2D dose at depth $z$ in water can be calculated by

$\mathrm{D}=\iint N_{i j} \cdot f_{i j}\left(\sigma_{1}, \sigma_{2}, w_{1}, w_{2}, x, y, z\right) \cdot D D D_{i}\left(E_{\text {beam }}, z\right) d i d j$

$i$ is the ordinal number of IES, i.e. the beam Bragg-peak is located at $i$ th IES which is related to the primary energy $E_{\text {beam }} . j$ is the ordinal number of pencil beam for each IES. $i j$ represent the $j$ th pencil beam for $i$ th IES. The depth dose distribution (DDD) profile and spot size (double Gaussian distribution) parameters at certain depth in water are the basic beam data in our clinical TPS.

\subsection{Validation of the software}

\subsubsection{Reconstruction algorithm verification}


In order to verify the accuracy of reconstruction algorithm, we compared the dose calculated by TPS with reconstructed 2D dose distribution using the same beam parameters. The mean local dose difference were calculated for proton and carbon ion plans, respectively. Ten carbon ion plans with 25 beams and ten proton plans with 25 beams were used for the algorithm verification. For each beam the comparison were done at three different depth, one is at the proximal edge of high dose region, another one is at the middle of high dose region and the third one is at the distal end of high dose region. All dose reconstruction used $1 \mathrm{~mm}$ grid size. The doses calculated by TPS with $2 \mathrm{~mm}$ or $3 \mathrm{~mm}$ grid size were interpolated to $1 \mathrm{~mm}$ grid size.

\subsubsection{Sensitivity of gamma pass rate to different deviations}

In order to investigate the sensitivity of gamma pass rate to spot size deviation, position deviation and particle number deviation. Different level of Gaussian-like random errors were manually added to the PBP plan parameters to generate modified plans. Then reconstructed dose based on modified plans were compared with the original plan dose calculated by TPS. During simulation, the spot size deviation levels were ranged from $1 \%$ to $34 \%$, the spot position deviation levels were ranged from $0.1 \mathrm{~mm}$ to $3 \mathrm{~mm}$ and the particle number deviation levels were ranged from $0.1 \%$ to $2 \%$. Deviation of spot size, spot position and particle number were investigated separately. The sigma value of the Gaussian like random error is obtained through the comparison of beam parameters between the PBP and the PBR of the 20 plans. The standard deviation of the spot size difference, spot position difference and particle number difference were used as the standard deviation in Gaussian distribution to generate random errors. The dose comparison for each beam was done at 3 
different depth, which were the same as the depth used for algorithm verification. Each deviation level was calculated 10 times for each beam to maintain the data stability. Gamma pass rate with $3 \mathrm{~mm} / 3 \%$ criteria was applied to evaluate the difference between the original plan dose and modified plan dose for carbon and proton beams respectively.

\subsubsection{Plan delivery verification using homemade software}

At last, actual plan delivery verification of the 20 plans were performed by using homemade software. The depth for actual plan delivery verification is the same as for algorithm validation. Spot size deviation in $\mathrm{x}$ and $\mathrm{y}$ direction $(\Delta \mathrm{Fx}, \Delta \mathrm{Fy})$, position deviation in $\mathrm{x}$ and $\mathrm{y}$ direction $(\Delta \mathrm{x}, \Delta \mathrm{y})$ and particle number deviation in each spot $(\Delta \mathrm{N})$ were also analyzed for each pencil beam.

\section{Results}

The user interface of homemade software and an example of verification is shown in Fig. 2. The software has the dose reconstruction and gamma analysis functions. Users can manually set the reconstruction resolution and depth in water and change the gamma analysis criteria. The reconstructed dose distribution can be exported as DICOM format. The software also can calculate the deviations of spot size, spot position and particle number for each beam between actual delivered value and planned value. These statistics can also be exported for further analysis. For the reconstruction of one dose slice, it takes about1040 seconds in personal laptop.

\section{Fig.2}

For the verification of dose reconstruction algorithm, the mean dose difference between the dose calculated by TPS and the dose reconstructed from the same parameters were 
shown in Table I. This small difference may be caused by the data interpolation of depth dose distribution during dose reconstruction and interpolation of dose grid size. We believe it is accurate enough for quality assurance using gamma analysis method with such small differences.

\section{Table.I}

For the sensitivity investigation, Fig. 3 shows the relationship between gamma pass rate and mean deviation level of spot size, spot position and particle number respectively. From Fig.3 (a) we can see that proton beams are more sensitive to spot size deviation, which is due to the bigger spot size of proton beams than that of carbon ion beams. First, for bigger spot size spot size deviation of one spot will affect more areas. Second, bigger spot size will result in sparse spot distribution, which can lead to less mutual compensation between spots especially for small or narrow areas. From Fig.3 (b) and (c) we can see that carbon ion beams are more sensitive to spot position deviation and particle number deviation, that is because the spot size of carbon ion beams are smaller than that of proton beams. It is obvious that for smaller spot size the same particle number deviation and absolute spot position deviation will result in bigger dose deviation in local area.

\section{Fig.3}

For the actual plan delivery verification results of the 20 treatment plans using homemade software, Gamma pass rate of comparison between dose reconstructed from to PBR and dose calculated by TPS is shown in Table II. The deviations of spot size, spot position and particle number between actual delivered value (PBR) and planned value are also listed in the table. Local dose normalization was used in gamma evaluation for an area with dose 
above $10 \%$ of the maximum dose. It reveals that the gamma pass rate for carbon ion beams and proton beams were almost $100 \%$ at present deviation level, which is consistent with the simulation results in Fig.3.

\section{Table.II}

\section{Discussion}

At present, all plan verification systems for proton and carbon ion radiotherapy are designed for pretreatment verification, which can't provide plan delivery information during treatment. Although all radiotherapy facilities have the beam application and monitoring system, it can only make sure all parameters are within their own tolerance and can't give quantitative dose information. It is very important to do plan verification of actual treatment to quantitatively show the dose differences between the dose actual delivered and the dose calculated by TPS. Several studies have using the plan CT for dose reconstruction based on $\log$ files to do the patient specific quality assurance ${ }^{21-24}$. The position deviation and MU deviation of pencil beams were analyzed for dose difference analysis. However, the spot size deviation of the pencil beam was not analyzed in these studies, which is also a nonnegligible reason affecting treatment accuracy.

In this study, we developed a verification software based on log files for scanning beam proton and carbon ion radiotherapy. The algorithm for dose reconstruction was validated by the good consistency between the dose calculated by TPS and the dose reconstructed from the same beam parameters. The relationship between gamma pass rate and different deviation levels were also investigated. Due to the smaller spot size of carbon ion beam than that of proton beam, the pass rate of carbon ion beams are more sensitive to spot 
position deviation and particle number deviation, and the pass rate of proton beam is more sensitive to spot size deviation.

For actual treatment verification using homemade software, Table II reveals that the gamma pass rate are almost $100 \%$ for both proton and carbon ion beams at present deviation level, which means our treatment system can accurately deliver plans generated by TPS. Although this method and homemade software can not verify the beam energy. We believe that the extracted beam energy is correct by default. Not only because of our comprehensive routine quality assurance that the beam energy is checked using peak-finder monthly, but also by the nature that the beam extraction needs perfect collaboration of the complicated accelerate system, bending magnet and extraction system. The consistency of Table II and Fig. 3 means that the relationship between gamma pass rate and different deviation level in Fig.3 are correct. In general, verification using homemade software do reveal the delivery errors during patient treatment, which means that our methods and software is feasible and reliable to do the plan delivery verification. Although the beam parameters are measured in the beam nozzle, they are converted to values at iso-center by a lookup table and stored in the log file. In order to ensure the accuracy of BAMS detectors and the look up table, routine quality assurance are carried out daily and weekly. During daily QA the beam position and spot size of a set of energies are checked by film. The transmission ion chambers in the BAMS is also calibrated by a farmer chamber everyday. The lookup table to convert the values measured in BAMS to values at iso-center is checked every week using film and MWPC.

We did 2D rather than $3 \mathrm{D}$ dose reconstruction to save calculation time. To do more 
sufficient verification one can chose several different depth at high dose region. The dose is reconstructed in water phantom rather than in patient $\mathrm{CT}$, because we can not get online CT or CBCT, which represent the actual patient anatomy during treatment. To do the reconstruction in plan CT can't show sufficiently accurate and more valuable information and time consuming. So the verification method in this study is only check the plan delivery accuracy of treatment system and the impacts of different beam parameters. In the future if we can get online $\mathrm{CT}$ or $\mathrm{CBCT}$, we will do the 3D dose reconstruction in the actual patient geometry for "in vivo" dose verification.

Other than the analysis of reconstructed dose distribution, the homemade software calculate the discrepancies of spot size, spot position and particle number. These results could be part of the daily performance check of the beam delivery system during patient treatment and can help to analyze the results of gamma evaluation. In the future, we will integrate the homemade software into the radiation oncology system, which will allow the immediate analysis right after the beam is irradiated. The results could be part of the treatment report for each patient.

\section{Conclusions}

We introduced a method and developed a software to do plan delivery verification based on $\log$ file for proton and carbon ion beam radiotherapy with pencil-beam scanning technique. Verification pass rate of carbon ion beam is more sensitive to spot position deviation and particle number deviation, and pass rate of proton beam is more sensitive to spot size deviation. Clinical validation results show that the homemade software for the treatment dose verification is feasible and reliable. Such tool can check the online performance of the 
proton and carbon ion delivery system and evaluate the impacts of beam parameter variations on the plan delivery accuracy, which will enhance current treatment verification processes.

\section{Abbreviations}

TPS: treatment planning system

LET: linear energy transfer

RBE: relative biological effectiveness

PET: positron emission tomography

IES: iso-energy slice

DIC: dynamic intensity control

BAMS: beam application and monitoring system

PBP: physical beam plan

PBR: physical beam record

DDD: depth dose distribution

DICOM: digital imaging and communications in medicine

QA: quality assurance

CT: computed tomography

CBCT: cone beam computed tomography

\section{Declarations}

\section{Acknowledgments}


Not applicable.

\section{Authors' contributions}

Jun Zhao designed the study and drafted the manuscript. Zhi Chen write the code.

Xainwei Wu, Ying Xing and Yongqiang Li participated in the data collection and analysis. All authors read and approved the final manuscript.

\section{Funding}

This study was supported by research funding from the National Natural Science

Foundation (11905035).

\section{Availability of data and materials}

The datasets used and analyzed during the current study are available upon reasonable request.

\section{Ethics approval and consent to participate}

All data were collected according to the guidelines of the Ethics Committee in Medical Research of the Fudan University Shanghai Cancer Center and Shanghai Proton and Heavy Ion Center.

\section{Consent for publication}

Not applicable.

\section{Competing interests}

The authors declare that they have no competing interests.

\section{Reference}

1. Jensen $\mathrm{AD}$, Poulakis $\mathrm{M}$, Nikoghosyan $\mathrm{AV}$, et al. High-LET radiotherapy for adenoid cystic carcinoma of the head and neck: 15 years' experience with raster-scanned carbon ion therapy. 
Radiotherapy and Oncology. 2016;118(2):272-280.

2. Fukahori M, Matsufuji N, Himukai T, et al. Estimation of late rectal normal tissue complication probability parameters in carbon ion therapy for prostate cancer. Radiotherapy and Oncology. 2016;118(1):136-140.

3. Eaton BR, Esiashvili N, Kim S, et al. Clinical outcomes among children with standard-risk medulloblastoma treated with proton and photon radiation therapy: a comparison of disease control and overall survival. International Journal of Radiation Oncology* Biology* Physics. 2016;94(1):133-138.

4. Kamada T, Tsujii H, Blakely EA, et al. Carbon ion radiotherapy in Japan: an assessment of 20 years of clinical experience. The Lancet Oncology. 2015;16(2):e93-e100.

5. Grün R, Friedrich T, Elsässer T, et al. Impact of enhancements in the local effect model (LEM) on the predicted RBE-weighted target dose distribution in carbon ion therapy. Physics in Medicine \& Biology. 2012;57(22):7261.

6. Schardt D, Elsässer T, Schulz-Ertner D. Heavy-ion tumor therapy: Physical and radiobiological benefits. Reviews of modern physics. 2010;82(1):383.

7. Schulz-Ertner D, Tsujii H. Particle radiation therapy using proton and heavier ion beams. Journal of clinical oncology. 2007;25(8):953-964.

8. Lomax A. Intensity modulated proton therapy and its sensitivity to treatment uncertainties 2: the potential effects of inter-fraction and inter-field motions. Physics in Medicine \& Biology. 2008;53(4):1043.

9. Kumagai M, Hara R, Mori S, et al. Impact of intrafractional bowel gas movement on carbon ion beam dose distribution in pancreatic radiotherapy. International Journal of Radiation Oncology* Biology* Physics. 2009;73(4):1276-1281.

10. Lomax AJ, Böhringer T, Bolsi A, et al. Treatment planning and verification of proton therapy using spot scanning: initial experiences. Medical physics. 2004;31(11):3150-3157.

11. Spielberger B, Krämer M, Kraft G. Three-dimensional dose verification with x-ray films in conformal carbon ion therapy. Physics in Medicine \& Biology. 2003;48(4):497.

12. Karger CP, Jäkel O, Hartmann GH, Heeg P. A system for three-dimensional dosimetric verification of treatment plans in intensity-modulated radiotherapy with heavy ions. Medical physics. 1999;26(10):2125-2132.

13. Arjomandy B, Sahoo N, Ciangaru G, Zhu R, Song X, Gillin M. Verification of patient-specific dose distributions in proton therapy using a commercial two-dimensional ion chamber array. Medical physics. 2010;37(11):5831-5837.

14. Zhu X, El Fakhri G. Proton therapy verification with PET imaging. Theranostics. 2013;3(10):731.

15. Bauer J, Unholtz D, Sommerer F, et al. Implementation and initial clinical experience of offline PET/CT-based verification of scanned carbon ion treatment. Radiotherapy and Oncology. 2013;107(2):218-226.

16. Polf JC, Avery S, Mackin DS, Beddar S. Imaging of prompt gamma rays emitted during delivery of clinical proton beams with a Compton camera: feasibility studies for range verification. Physics in Medicine \& Biology. 2015;60(18):7085.

17. Sportelli G, Belcari N, Camarlinghi N, et al. First full-beam PET acquisitions in proton therapy with a modular dual-head dedicated system. Physics in Medicine \& Biology. 2013;59(1):43.

18. Parodi K, Mairani A, Sommerer F. Monte Carlo-based parametrization of the lateral dose spread for clinical treatment planning of scanned proton and carbon ion beams. Journal of radiation research. 
2013;54(suppl_1):i91-i96.

19. Bellinzona VE, Ciocca M, Embriaco A, et al. On the parametrization of lateral dose profiles in proton radiation therapy. Physica medica :PM : an international journal devoted to the applications of physics to medicine and biology : official journal of the Italian Association of Biomedical Physics. Jul 2015;31(5):484-492.

20. Low DA, Harms WB, Mutic S, Purdy JA. A technique for the quantitative evaluation of dose distributions. Medical physics. 1998;25(5):656-661.

21. Meier G, Besson R, Nanz A, Safai S, Lomax AJ. Independent dose calculations for commissioning, quality assurance and dose reconstruction of PBS proton therapy. Physics in medicine and biology. Apr 7 2015;60(7):2819-2836.

22. Belosi MF, van der Meer R, Garcia de Acilu Laa P, Bolsi A, Weber DC, Lomax AJ. Treatment log files as a tool to identify treatment plan sensitivity to inaccuracies in scanned proton beam delivery. Radiotherapy and oncology : journal of the European Society for Therapeutic Radiology and Oncology. Dec 2017;125(3):514-519.

23. Li H, Sahoo N, Poenisch F, et al. Use of treatment log files in spot scanning proton therapy as part of patient-specific quality assurance. Medical physics. Feb 2013;40(2):021703.

24. Zhu XR, Li Y, Mackin D, et al. Towards effective and efficient patient-specific quality assurance for spot scanning proton therapy. Cancers. Apr 10 2015;7(2):631-647.

\section{Figure legends}

Fig. 1. Diagram of the beam application and monitoring system (BAMS) which consist of three ion chambers (IC and IM) and two multi-ware proportional chambers (MW). Spot size, spot position and particle number in each spot is measured by the BAMS and are recorded in log file.

Fig. 2. User interface of the homemade software and an example of verification. Subplot titled "PBR" is the reconstructed dose in water-phantom at selected depth. Subplot titled "Vx Dose" is the dose calculated by TPS imported to the software. Subplot titled "pass rate" shows the gamma pass rate and spots do not pass the gamma criteria, which are in red color. Subplot titled "gamma histogram" shows the gamma index distribution. Left part are the buttons for data import and dose reconstruction and edit boxes for setting dose reconstruction and gamma analysis parameters. Right part are edit boxes for analysis results and buttons for data export.

Fig.3. Variation of gamma pass rate with the changes of spot size deviation level, spot position deviation level and particle number deviation level respectively. $3 \mathrm{~mm} / 3 \%$ criteria was used in Gamma analysis. 
Figures

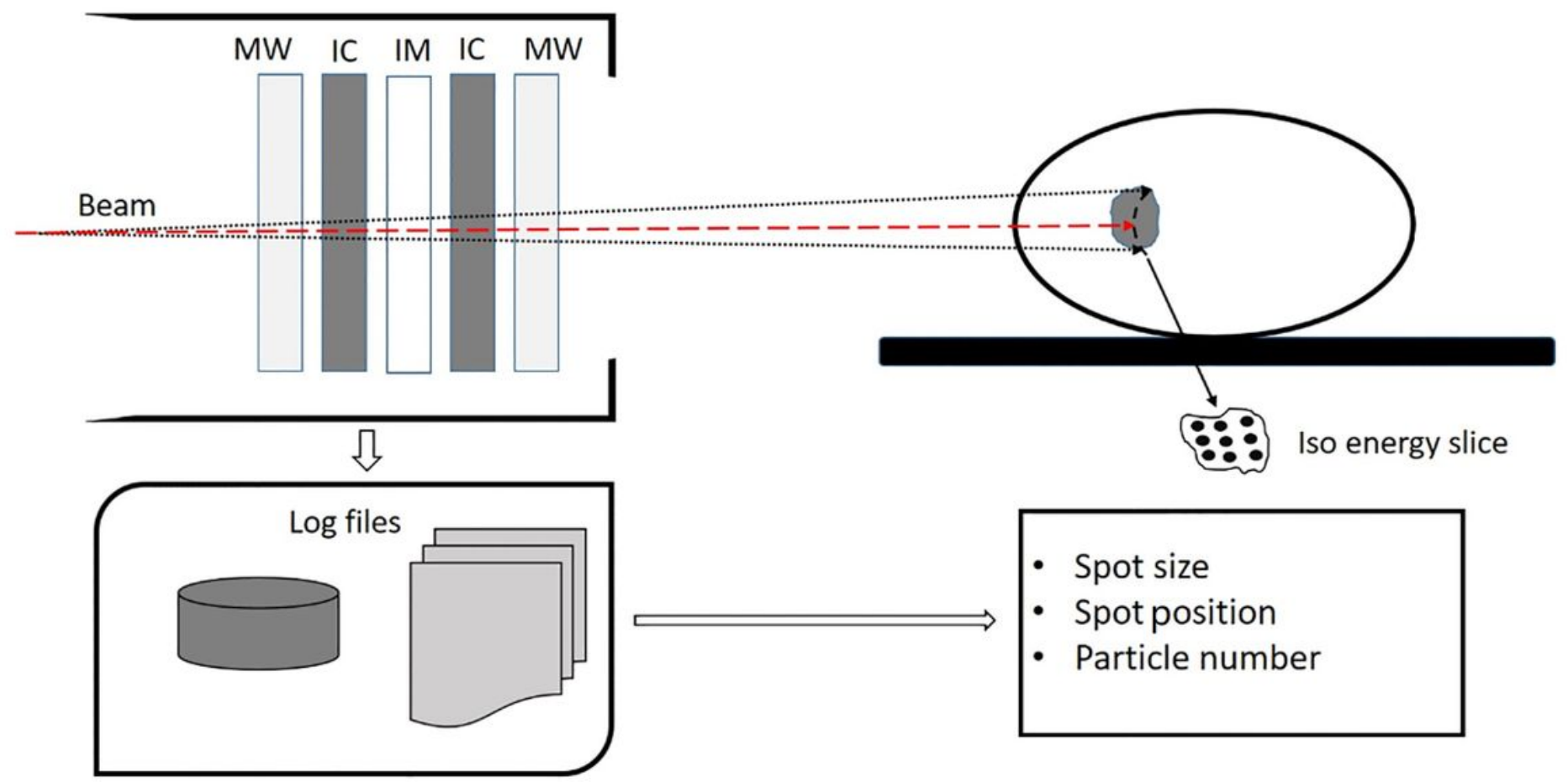

Figure 1

Diagram of the beam application and monitoring system (BAMS) which consist of three ion chambers (IC and $\mathrm{IM}$ ) and two multi-ware proportional chambers (MW). Spot size, spot position and particle number in each spot is measured by the BAMS and are recorded in log file. 


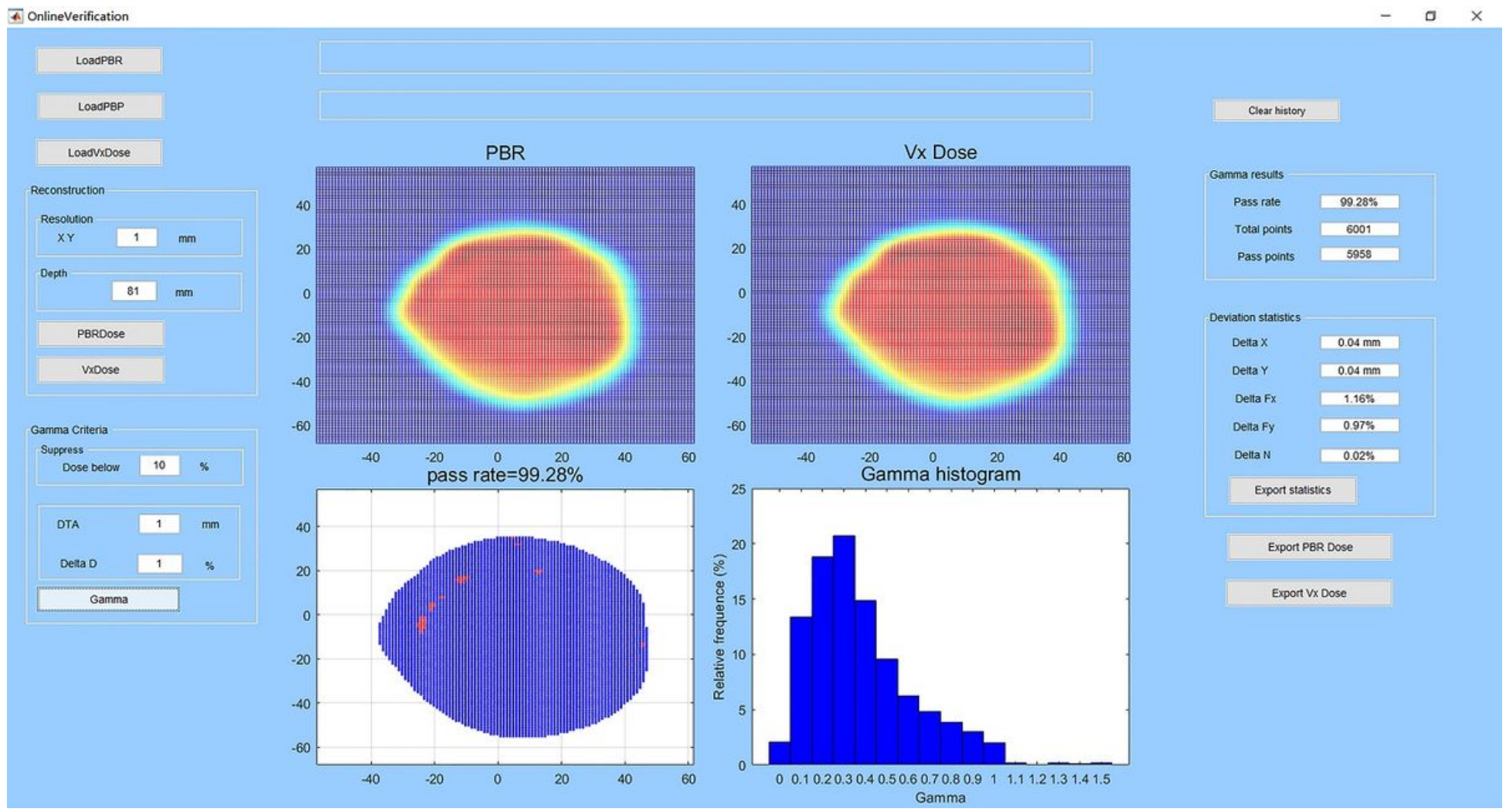

Figure 2

User interface of the homemade software and an example of verification. Subplot titled "PBR" is the reconstructed dose in water-phantom at selected depth. Subplot titled "Vx Dose" is the dose calculated by TPS imported to the software. Subplot titled "pass rate" shows the gamma pass rate and spots do not pass the gamma criteria, which are in red color. Subplot titled "gamma histogram" shows the gamma index distribution. Left part are the buttons for data import and dose reconstruction and edit boxes for setting dose reconstruction and gamma analysis parameters. Right part are edit boxes for analysis results and buttons for data export. 
(a)

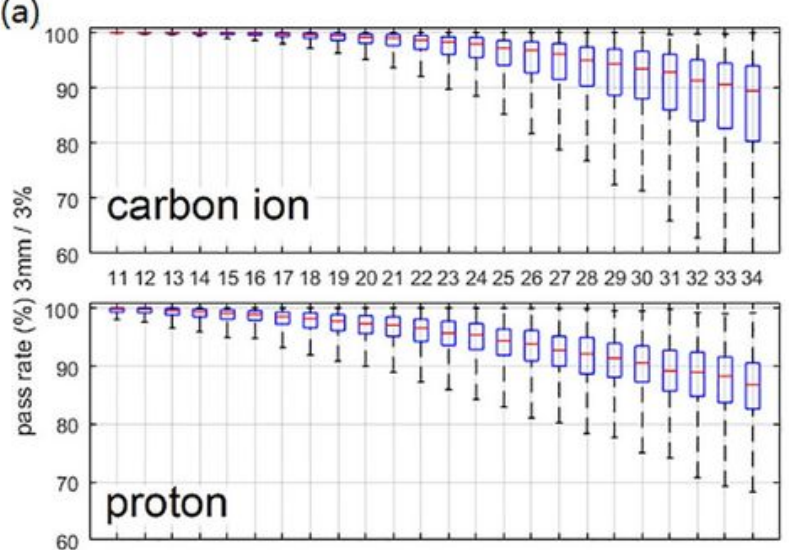

111213141516171819202122232425262728293031323334 spot size deviation (\%)

(b)

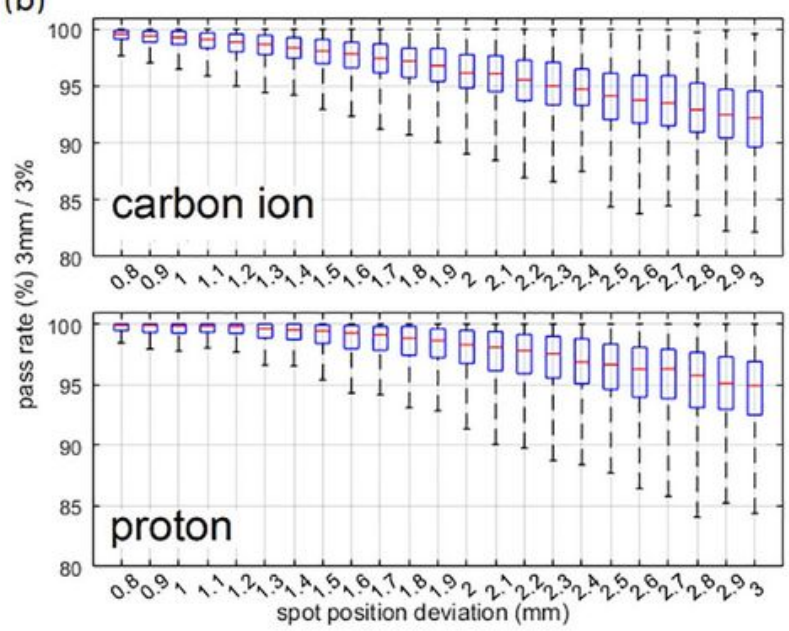

(c)

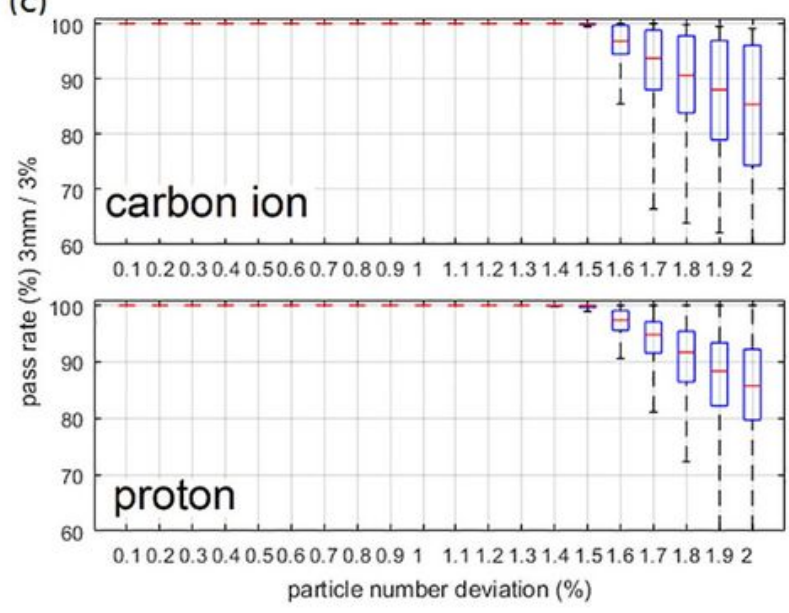

Figure 3

Variation of gamma pass rate with the changes of spot size deviation lev el, spot position deviation level and particle number deviation level respectively. $3 \mathrm{~mm} / 3 \%$ criteria was used in Gamma analysis.

\section{Supplementary Files}


This is a list of supplementary files associated with this preprint. Click to download.

- table2.JPG 